PROCEEDINGS OF THE

AMERICAN MATHEMATICAL SOCIETY

Volume 132, Number 3, Pages 891-898

S 0002-9939(03)07221-6

Article electronically published on October 21, 2003

\title{
HOMOLOGY EQUIVALENCES INDUCING AN EPIMORPHISM ON THE FUNDAMENTAL GROUP AND QUILLEN'S PLUS CONSTRUCTION
}

\author{
JOSÉ L. RODRÍGUEZ AND DIRK SCEVENELS
}

(Communicated by Paul Goerss)

\begin{abstract}
Quillen's plus construction is a topological construction that kills the maximal perfect subgroup of the fundamental group of a space without changing the integral homology of the space. In this paper we show that there is a topological construction that, while leaving the integral homology of a space unaltered, kills even the intersection of the transfinite lower central series of its fundamental group. Moreover, we show that this is the maximal subgroup that can be factored out of the fundamental group without changing the integral homology of a space.
\end{abstract}

\section{INTRODUCTION}

As explained in [8, 9], Bousfield's $H \mathbb{Z}$-localization $X_{H \mathbb{Z}}$ of a space $X$ ([2]) is homotopy equivalent to its localization with respect to a map of classifying spaces $B f: B \mathcal{F}_{1} \rightarrow B \mathcal{F}_{2}$ induced by a certain homomorphism $f: \mathcal{F}_{1} \rightarrow \mathcal{F}_{2}$ between free groups. This means that a space $X$ is $H \mathbb{Z}$-local if and only if the induced map $B f^{*}: \operatorname{map}\left(B \mathcal{F}_{2}, X\right) \rightarrow \operatorname{map}\left(B \mathcal{F}_{1}, X\right)$ is a weak homotopy equivalence. Moreover, the effect of $B f$-localization on the fundamental group produces precisely the group-theoretical $H \mathbb{Z}$-localization (i.e., $f$-localization) of the fundamental group, i.e., $\pi_{1} L_{B f} X \cong L_{f}\left(\pi_{1} X\right) \cong\left(\pi_{1} X\right)_{H \mathbb{Z}}$ for all spaces $X$.

A universal acyclic space for $H \mathbb{Z}$-localization (i.e., $B f$-localization), in the sense of Bousfield (4]), was studied by Berrick and Casacuberta in 1]. They show that nullification with respect to such a universal acyclic space coincides with Quillen's plus construction $X^{+}$for a space $X$. Moreover, this universal acyclic space can be taken to be a two-dimensional Eilenberg-Mac Lane space $K(A(f), 1)$, where $A(f)$ is a locally free, universal $f$-acyclic group, in the sense of [12. The effect of $K(A(f), 1)$-nullification on the fundamental group is precisely given by its $A(f)$ nullification. This $A(f)$-nullification factors out the perfect radical of a group, i.e., the maximal perfect subgroup, which can be obtained as the intersection of the (transfinite) derived series of the group ([7]).

The intersection $\Gamma G$ of the transfinite lower central series of a group $G$ is also a radical, and, as observed by Bousfield ([3]), it is in fact the maximal $G$-perfect normal subgroup of $G$ (where a normal subgroup $H$ of $G$ is called $G$-perfect if

Received by the editors July 29, 2002.

2000 Mathematics Subject Classification. Primary 55P60, 55N15; Secondary 18A20, 18A40.

The first author was supported by the Spanish DGES grant PB97-0202. 
$H=[H, G])$. As explained in [7], there is an epireflection that corresponds to this radical $\Gamma G$. By adapting the methods used by Berrick and Casacuberta in [1, we describe in Theorem 2.5 an epimorphism $g$ such that $L_{g} G \cong G / \Gamma G$ for all groups $G$.

We further construct a localization functor of topological spaces that is intermediate between Quillen's plus construction and Bousfield's homological localization, and induces localization with respect to $g$ on the fundamental group. More precisely, we describe in Theorem 2.9 a map $\varphi$ such that $\varphi$-localization of spaces factors out the intersection of the transfinite lower central series of the fundamental group, while preserving the integral homology of the space.

We furthermore show that this is in some sense the best possible result. Indeed, we show in Proposition 3.2 that the maximal subgroup that can be factored out of the fundamental group without altering the integral homology of a space is precisely the intersection of the transfinite lower central series of the fundamental group.

Finally, we turn our attention to the question whether there exists a localization functor of spaces that induces localization with respect to a universal epimorphic $H \mathbb{Z}$-equivalence of groups (in the sense of [12]) on the fundamental group, without changing the integral homology of a space. In fact, we show in Proposition 3.3 that the answer to this question is affirmative if and only if, for all groups $G$, the kernel of the $H \mathbb{Z}$-localization homomorphism $G \rightarrow G_{H \mathbb{Z}}$ coincides with $\Gamma G$.

\section{Preliminaries}

For the convenience of the reader, we recall some terminology and basic facts about localization with respect to a given continuous map (see e.g. [4], [8]). Given a map $f: A \rightarrow B$, a space $X$ is called $f$-local if the induced map

$$
f^{*}: \operatorname{map}(B, X) \rightarrow \operatorname{map}(A, X)
$$

is a weak homotopy equivalence. For every space $X$ there is a map $l_{X}: X \rightarrow$ $L_{f} X$, which is initial among all maps from $X$ into $f$-local spaces. $L_{f}$ is called the localization functor with respect to $f$. A map $\phi$ is called an $f$-equivalence if $L_{f} \phi$ is a homotopy equivalence. Furthermore, a space $X$ is called $f$-acyclic if $L_{f} X$ is contractible. In the special case where $f$ is of the form $f: A \rightarrow *$, the $f$-localization of a space $X$ is also denoted by $P_{A} X$ and it is called the $A$-nullification of $X$. The localization class of $f$, denoted by $\langle f\rangle$, is defined as the collection of all maps $g$ such that $L_{g}$ is naturally equivalent to $L_{f}$. When $f$ is of the form $f: A \rightarrow *$, the class of $f$ is simply denoted by $\langle A\rangle$ and is called the nullification class of $A$. One says that $\langle f\rangle \leq\langle g\rangle$ if and only if there is a natural transformation of localization functors $L_{f} \rightarrow L_{g}$. Recall that this is equivalent to every $g$-local space being $f$-local, to every $f$-equivalence being a $g$-equivalence, or to $f$ being a $g$-equivalence. As shown by Bousfield in [4, the collection of localization functors with respect to maps is a small-complete lattice for this partial order relation. Note that a space $X$ is $f$ acyclic if and only if $\langle X\rangle \leq\langle f\rangle$. Furthermore, in [4 Bousfield proved that every localization class has a best possible approximation by a nullification class. More precisely, for any map $f$, there is a maximal nullification class $\langle A(f)\rangle$ that is smaller than $\langle f\rangle$ in the lattice of localization classes. The space $A(f)$ is called a universal $f$-acyclic space, since a space $X$ is $f$-acyclic if and only if $X$ is $A(f)$-acyclic. 
An important algebraic tool in studying localization with respect to a given map is given by its discrete analogue in the category of groups (see e.g. [4, 5]). For a given group homomorphism $f: A \rightarrow B$, a group $G$ is called $f$-local if the induced map of sets

$$
f^{*}: \operatorname{Hom}(B, G) \rightarrow \operatorname{Hom}(A, G)
$$

is a bijection. For every group $G$ there is a homomorphism $l_{G}: G \rightarrow L_{f} G$, which is initial among all homomorphisms from $G$ into $f$-local groups. This allows us to introduce the localization functor $L_{f}$ with respect to $f$. In an obvious way, one speaks of $f$-equivalences and $f$-acyclic groups, and, if $f$ is of the form $f$ : $A \rightarrow 1$, of $A$-nullification. It also makes sense to speak of the localization class of a homomorphism $f$, denoted by $\langle f\rangle$, which in the special case when $f$ is of the form $f: A \rightarrow 1$, is simply denoted by $\langle A\rangle$ and is called the nullification class of $A$. Furthermore, the collection of all localization classes of homomorphisms is again a small-complete lattice for an obvious partial order relation. In [12] it was proved that every localization class of a homomorphism has a best possible approximation by a nullification class and by the localization class of an epimorphism. More precisely, for any homomorphism $f$, there is a maximal nullification class $\langle A(f)\rangle$ and a maximal class $\langle\mathcal{E}(f)\rangle$ where $\mathcal{E}(f)$ is an epimorphism such that

$$
\langle A(f)\rangle \leq\langle\mathcal{E}(f)\rangle \leq\langle f\rangle .
$$

The group $A(f)$ is called a universal $f$-acyclic group, since a group $G$ is $f$-acyclic if and only if $G$ is $A(f)$-acyclic. Accordingly, $\mathcal{E}(f)$ is called a universal epimorphic $f$-equivalence, since an epimorphism $g$ is an $f$-equivalence if and only if $g$ is an $\mathcal{E}(f)$-equivalence.

It was shown in [12] (see also [7]) that to any localization class $\langle f\rangle$ of an epimorphism $f$, there is associated a radical $R_{f}$ on the category of groups such that $L_{f} G \cong G / R_{f} G$ for all groups $G$. (Recall that a radical $R$ is a functor that assigns to every group $G$ a normal subgroup $R G$ in such a way that every homomorphism $G \rightarrow K$ restricts to $R G \rightarrow R K$ and such that $R(G / R G)=1$.) In fact, there is a bijective correspondence between epireflections (i.e., idempotent functors $L$ on the category of groups for which $G \rightarrow L G$ is an epimorphism for any group $G$ ) and radicals. Furthermore, if the class $\langle f\rangle$ is actually a nullification class, then the associated radical $R_{f}$ is idempotent, meaning that $R_{f} R_{f} G=R_{f} G$ for all groups $G$.

\section{A UNIVERSAL EPIMORPHIC $H \mathbb{Z}$-MAP}

It is well known that Bousfield's $H \mathbb{Z}$-localization $X_{H \mathbb{Z}}$ of a space $X$ ([2]) is homotopy equivalent to its localization with respect to a map $B f: B \mathcal{F}_{1} \rightarrow B \mathcal{F}_{2}$ induced by a certain homomorphism $f: \mathcal{F}_{1} \rightarrow \mathcal{F}_{2}$ between free groups ([8], [9]). In fact, the homomorphism $f$ can be taken to be the free product of a set of representatives of isomorphism classes of homomorphisms between countable, free groups inducing an isomorphism on the first integral homology group. Furthermore, the effect of $B f$-localization on the fundamental group is to produce its $f$-localization, which coincides with the group-theoretical $H \mathbb{Z}$-localization of the fundamental group. In [1, Proposition 4.2] the authors show that a universal $f$-acyclic group $A(f)$ (in the sense of [12]) can be taken to be the free product of a set of representatives of all isomorphism classes of countable, locally free, perfect groups. The key lemma here is a result due to Heller ([10]), which states that for every element $x$ in any perfect 
group $P$, there exists a countable, locally free, perfect group $D$ and a homomorphism $D \rightarrow P$ containing $x$ in its image. We can similarly show the following lemma. Recall that a normal subgroup $H$ of a group $G$ is called $G$-perfect if $H=[H, G]$. In fact, any group $G$ has a maximal $G$-perfect subgroup, which we denote by $\Gamma G$, and that can be obtained as the intersection of the transfinite lower central series of $G$ (see [3]).

Lemma 2.1. Let $A$ be any group with an A-perfect normal subgroup $K$. Let $z$ be any element in $K$. Then there exist a countable, locally free group $D$ and a homomorphism $\psi: D \rightarrow A$ such that $z$ belongs to the image of some element in $\Gamma D$.

Proof. Since $K=[K, A]$ we can express $z$ as a finite product of commutators $z=\Pi_{i}\left[k_{i}, a_{i}\right]$ where $k_{i} \in K, a_{i} \in A$. Now again, for each $k_{i}$, we can find finitely many elements $k_{i, l} \in K, a_{i, l} \in A$, such that $k_{i}=\Pi_{l}\left[k_{i, l}, a_{i, l}\right]$. Similarly for $k_{i, l}$ we have $k_{i, l}=\Pi_{m}\left[k_{i, l, m}, a_{i, l, m}\right]$, with $k_{i, l, m} \in K$ and $a_{i, l, m} \in A$, etc. For each element appearing in these commutator decompositions we choose a free generator, which we denote by $x, x_{i}, y_{i}, x_{i, l}, y_{i, l}, x_{i, l, m}, y_{i, l, m}$, etc. Then we define $D$ as the following direct limit of free groups:

$$
\operatorname{Fr}(x) \stackrel{\psi_{0}}{\longrightarrow} \operatorname{Fr}\left(x_{i}, y_{i}\right) \stackrel{\psi_{1}}{\longrightarrow} \operatorname{Fr}\left(x_{i, l}, y_{i, l}, y_{i}\right) \stackrel{\psi_{2}}{\longrightarrow} \operatorname{Fr}\left(x_{i, l, m}, y_{i, l, m}, y_{i, l}, y_{i}\right) \stackrel{\psi_{3}}{\longrightarrow} \cdots
$$

where the $\psi_{j}$ are defined as the decompositions above, that is, $\psi_{0}(x)=\Pi_{i}\left[x_{i}, y_{i}\right]$; $\psi_{1}\left(x_{i}\right)=\Pi_{l}\left[x_{i, l}, y_{i, l}\right]$, and the identity on the $y_{i}$ 's; $\psi_{2}\left(x_{i, l}\right)=\Pi_{m}\left[x_{i, l, m}, y_{i, l, m}\right]$, and the identity on the other generators, etc. Observe that $D$ is countable and locally free. The obvious homomorphism $\psi: D \rightarrow A$ is defined as $\psi(x)=z, \psi\left(x_{i}\right)=k_{i}$, $\psi\left(y_{i}\right)=a_{i}, \psi\left(x_{i, l}\right)=k_{i, l}, \psi\left(y_{i, l}\right)=a_{i, l}$, etc. It is clear by construction that $x \in \Gamma D$ and $\psi(x)=z$ as required.

Proposition 2.2. Let $G$ be any group. Then the following assertions are equivalent:

(i) For every group $A$ and every A-perfect normal subgroup $K$ of $A$, the restriction of any homomorphism $A \rightarrow G$ to $K$ is trivial;

(ii) For every countable, locally free group $A$ and every $A$-perfect normal subgroup $K$ of $A$, the restriction of any homomorphism $A \rightarrow G$ to $K$ is trivial;

(iii) For every countable, locally free group $A$, the restriction of any homomorphism $A \rightarrow G$ to $\Gamma A$ is trivial.

The subgroup $\Gamma G$ actually defines a radical on the category of groups, and, hence, by [7], the assignation $G \rightarrow L G=G / \Gamma G$ is an epireflection. Our aim is to show that this epireflection is singly generated. More precisely, we want to exhibit a homomorphism $g$ such that $L_{g} G \cong L G=G / \Gamma G$ for any group $G$. Moreover, by 12 we know that it is possible to choose $g$ to be an epimorphism.

Let $\Lambda$ be a set of representatives of isomorphism classes of countable, locally free groups. If $\mathcal{D}^{\prime}$ denotes the normal closure of $\bigcup_{\Lambda} \Gamma D$ in the free product $\mathcal{D}$ of all groups $D$ in $\Lambda$, which we denote by $\operatorname{Fr}_{\Lambda} D$, then we have a short exact sequence (cf. [11, Exercise 6.2.5])

$$
\mathcal{D}^{\prime} \longmapsto \mathcal{D}=\operatorname{Fr}_{\Lambda} D \rightarrow \mathcal{D} / \mathcal{D}^{\prime} \cong \operatorname{Fr}_{\Lambda}(D / \Gamma D),
$$

where $\operatorname{Fr}_{\Lambda}(D / \Gamma D)$ denotes the free product of all groups $D / \Gamma D$ for which (the isomorphism class of) $D$ belongs to $\Lambda$. 
Proposition 2.3. Localization with respect to the epimorphism

$$
h: \mathcal{D} \rightarrow \mathcal{D} / \mathcal{D}^{\prime}
$$

given in (1) satisfies $L_{h} G \cong G / \Gamma G$ for all groups $G$.

Proof. Observe that a group $G$ is $f$-local for any given epimorphism $f: A \rightarrow B$ if and only if the restriction of any homomorphism $A \rightarrow G$ to the kernel of $f$ is trivial. The proof is now completed by using Proposition 2.2.

Note that we can partition $\Lambda$ into $\Lambda_{1}$, containing all the representatives of the isomorphism classes of countable, locally free, perfect groups, and its complement $\Lambda_{1}^{c}$. We then can write

$$
\begin{aligned}
\langle h\rangle & =\left\langle\operatorname{Fr}_{\Lambda_{1}}(D \rightarrow D / \Gamma D)\right\rangle *\left\langle\operatorname{Fr}_{\Lambda_{1}^{c}}(D \rightarrow D / \Gamma D)\right\rangle \\
& =\left\langle\operatorname{Fr}_{\Lambda_{1}}(D \rightarrow 1)\right\rangle *\left\langle\operatorname{Fr}_{\Lambda_{1}^{c}}(D \rightarrow D / \Gamma D)\right\rangle \\
& =\langle\mathcal{F}\rangle *\left\langle\operatorname{Fr}_{\Lambda_{1}^{c}}(D \rightarrow D / \Gamma D)\right\rangle,
\end{aligned}
$$

where $\mathcal{F}$ is the universal $H \mathbb{Z}$-acyclic group defined in 1]. (Here we denote by $\left\langle f_{1}\right\rangle *\left\langle f_{2}\right\rangle$ the least upper bound of the classes $\left\langle f_{1}\right\rangle$ and $\left\langle f_{2}\right\rangle$ in the lattice of localization classes, and we have used the fact that the free product $f_{1} * f_{2}$ is a representative of this least upper bound.)

However, it is possible to give another description of $\langle h\rangle$, which will be more useful later on. Indeed, note that, if $f_{1}: A \rightarrow B$ is an epimorphism, and $f_{2}: B \rightarrow C$ is any homomorphism, then $\left\langle f_{2} \circ f_{1}\right\rangle=\left\langle f_{1} * f_{2}\right\rangle=\left\langle f_{1}\right\rangle *\left\langle f_{2}\right\rangle$. This enables us to prove the following preliminary result.

Lemma 2.4. Let $f: A \rightarrow B$ be any epimorphism. Then $\langle f\rangle=\left\langle l_{A}\right\rangle$, where $l_{A}: A \rightarrow$ $L_{f} A$ denotes the localization homomorphism.

Proof. Since $f$ is an epimorphism, we infer from [12, Theorem 2.1] that $l_{A}$ is an epimorphism. Hence,

$$
\langle f\rangle=\langle f\rangle *\left\langle l_{B}\right\rangle=\left\langle l_{B} \circ f\right\rangle=\left\langle L_{f} f \circ l_{A}\right\rangle=\left\langle L_{f} f\right\rangle *\left\langle l_{A}\right\rangle=\left\langle l_{A}\right\rangle .
$$

The above lemma now proves the following alternative description of $\langle h\rangle$.

Theorem 2.5. Consider the natural homomorphism $g: \mathcal{D} \rightarrow \mathcal{D} / \Gamma \mathcal{D}$, where $\mathcal{D}$ is as defined in (1). Then $\langle g\rangle=\langle h\rangle$, where $h$ is defined in (1). In other words, $L_{g} G \cong G / \Gamma G$ for all groups $G$.

In fact, the homomorphism $g$ is a "universal epimorphic $H \mathbb{Z}$-map", as we next show. Recall from [3] that a group homomorphism $g$ is called an $H \mathbb{Z}$-map if $H_{1}(g)$ (i.e., the homomorphism induced by $g$ on the first integral homology group) is an isomorphism and $H_{2}(g)$ is an epimorphism. We first need a characterization of the epimorphisms that are $H \mathbb{Z}$-maps (cf. [3]).

Lemma 2.6. Let $h$ be an epimorphism $h: A \rightarrow B$ with kernel $K$. Then $h$ is an $H \mathbb{Z}$-map if and only if $K$ is A-perfect.

Proof. This is an obvious consequence of the 5 -term exact sequence

$$
H_{2}(A) \rightarrow H_{2}(B) \rightarrow K /[K, A] \rightarrow H_{1}(A) \rightarrow H_{1}(B) \rightarrow 0 .
$$

Proposition 2.7. Let $G$ be any group and let $g: \mathcal{D} \rightarrow \mathcal{D} / \Gamma \mathcal{D}$, where $\mathcal{D}$ is as defined in (11). The homomorphism $G \rightarrow L_{g} G \cong G / \Gamma G$ is terminal among all epimorphic $H \mathbb{Z}$-maps going out of $G$. 
Observe that the epimorphism $g$ that we have constructed is not a universal epimorphic $H \mathbb{Z}$-equivalence (in the sense of [12]). Indeed, there are "more" epimorphic $H \mathbb{Z}$-equivalences than epimorphic $H \mathbb{Z}$-maps. However, if we denote by $A(f)$, resp. $\mathcal{E}(f)$ a universal $H \mathbb{Z}$-acyclic group, resp. a universal epimorphic $H \mathbb{Z}$-equivalence, then there are natural homomorphisms

$$
G \rightarrow P_{A(f)} G \rightarrow L_{g} G \cong G / \Gamma G \rightarrow L_{\mathcal{E}(f)} G \rightarrow G_{H \mathbb{Z}},
$$

for any group $G$, where $G \rightarrow L_{\mathcal{E}(f)} G \rightarrow G_{H \mathbb{Z}}$ is an epi-mono factorization. Moreover, for many groups $G$ (e.g., finite groups, nilpotent groups, or more generally, groups for which the lower central series stabilizes), we have isomorphisms $L_{g} G \cong G / \Gamma G \cong L_{\mathcal{E}(f)} G \cong G_{H \mathbb{Z}}($ cf. [3] $)$.

We now want to realize localization with respect to $g: \mathcal{D} \rightarrow \mathcal{D} / \Gamma \mathcal{D}$ topologically, by exhibiting a localization functor of spaces that induces $g$-localization on the fundamental group and that is intermediate between Quillen's plus construction and Bousfield's $H \mathbb{Z}$-localization (and, hence, does not change the integral homology of a space).

We will need the following proposition, which is similar to results obtained in [5] and $[\underline{6}$.

Proposition 2.8. Let $\psi: A \rightarrow B$ be any map that induces an epimorphism $\psi_{*}=$ $\pi_{1}(\psi): \pi_{1}(A) \rightarrow \pi_{1}(B)$ and suppose that $A$ is a $C W$-complex of dimension at most two. Then $\psi$-localization of spaces is $\pi_{1}$-compatible, i.e.,

$$
\pi_{1}\left(L_{\psi} X\right) \cong L_{\psi_{*}}\left(\pi_{1} X\right)
$$

for all spaces $X$.

Proof. For any space $X$, the map $X \rightarrow L_{\psi} X$ is a $\psi$-equivalence. Hence, by [5, Proposition 3.3], the induced homomorphism $\pi_{1}(X) \rightarrow \pi_{1}\left(L_{\psi} X\right)$ is a $\psi_{*^{-}}$ equivalence. Moreover, we claim that $\pi_{1}\left(L_{\psi} X\right)$ is $\psi_{*}$-local. To see this, it suffices to prove that the restriction of any homomorphism $\ell: \pi_{1} A \rightarrow \pi_{1}\left(L_{\psi} X\right)$ to ker $\psi_{*}$ is trivial. However, since the dimension of $A$ is at most two, there exists a map $\xi: A \rightarrow L_{\psi} X$ inducing $\ell$ on the fundamental group. Since $L_{\psi} X$ is $\psi$-local, we infer that there exists a map $\chi: B \rightarrow L_{\psi} X$ such that $\chi \circ \psi \simeq \xi$, which implies that the restriction $\ell\left|\operatorname{ker} \psi_{*}=\left(\pi_{1}(\chi) \circ \psi_{*}\right)\right| \operatorname{ker} \psi_{*}$ is trivial.

Now choose a two-dimensional CW-complex $M \mathcal{D}$ such that $\pi_{1} M \mathcal{D}=\mathcal{D}$. Attach 2-cells to $M \mathcal{D}$, thereby obtaining a map $i: M \mathcal{D} \hookrightarrow C$ which induces

$$
g: \mathcal{D} \rightarrow \mathcal{D} / \Gamma \mathcal{D}
$$

on the fundamental group. We then obtain a diagram

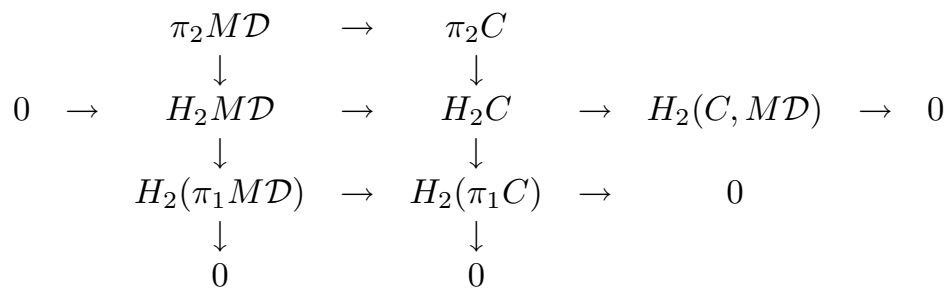

Moreover, since $H_{2}\left(\pi_{1} C\right)=H_{2}(\mathcal{D} / \Gamma \mathcal{D})=0$, we infer that $H_{2} C$ is an epimorphic image of $\pi_{2} C$. This means that we can kill $\mathrm{H}_{2} C$ by attaching 3 -cells to $C$, through 
a map $j: C \rightarrow C^{\prime}$. It is now easily verified that the composition $\varphi$ of

$$
M \mathcal{D} \stackrel{i}{\longrightarrow} C \stackrel{j}{\longrightarrow} C^{\prime}
$$

is an integral homology equivalence and that $\varphi$ induces the homomorphism $g: \mathcal{D} \rightarrow$ $\mathcal{D} / \Gamma \mathcal{D}$ on the fundamental group (cf. [2, Lemma 6.1]).

Theorem 2.9. Let $\varphi: M \mathcal{D} \rightarrow C^{\prime}$ be the composition given in (2). Then $\varphi$ localization of spaces induces g-localization on the fundamental group. Moreover, for any space $X$, there are natural maps

$$
X \rightarrow X^{+} \rightarrow L_{\varphi} X \rightarrow X_{H \mathbb{Z}}
$$

Proof. Since $M \mathcal{D}$ is a two-dimensional CW-complex and since $\varphi$ induces an epimorphism on the fundamental group, we know by Proposition 2.8 that $\varphi$-localization is $\pi_{1}$-compatible, so that it induces $g$-localization on the fundamental group. The second claim is obvious, since $\varphi$ is clearly an integral homology equivalence, the perfect radical of $\pi_{1}\left(L_{\varphi} X\right)$ being trivial (cf. 1]).

To see that the natural maps given in Theorem 2.9 are not equivalences in general, and thus that we have constructed a functor which is really different from Quillen's plus construction and from $H \mathbb{Z}$-localization, observe the following. On the one hand,

$$
L_{\varphi}\left(S^{1} \vee S^{1}\right) \simeq S^{1} \vee S^{1} \simeq\left(S^{1} \vee S^{1}\right)^{+} .
$$

Indeed, the fact that $\mathbb{Z} * \mathbb{Z}$ is $g$-local implies that $S^{1} \vee S^{1}$ is $\varphi$-local. On the other hand, $\pi_{1}\left(L_{\varphi} B \Sigma_{3}\right) \cong L_{g}\left(\pi_{1} B \Sigma_{3}\right) \cong \mathbb{Z} / 2$, while $\pi_{1} B \Sigma_{3}^{+} \cong P_{A(f)}\left(\pi_{1} B \Sigma_{3}\right) \cong \Sigma_{3}$.

\section{Homology EQUiVAlENCES INDUCING AN EPIMORPHISM ON THE FUNDAMENTAL GROUP}

In this section we want to explore some immediate consequences of our results. In particular, we want to show that there are some restrictions on (integral) homology equivalences that induce an epimorphism on the fundamental group.

Proposition 3.1. Let $\psi: X \rightarrow Y$ be an integral homology equivalence of spaces such that the induced homomorphism $f=\pi_{1} \psi$ is an epimorphism. Then $R_{f} G \subset \Gamma G$ for all groups $G$, where $R_{f}$ denotes the radical associated to the epireflection class $\langle f\rangle$.

Proof. By hypothesis we know that $f$ is an epimorphic $H \mathbb{Z}$-map. Hence, $\langle f\rangle \leq$ $\langle g\rangle$, where $g$ denotes the universal epimorphic $H \mathbb{Z}$-map of Theorem 2.5. Hence, there are natural homomorphisms $G \rightarrow L_{f} G \cong G / R_{f} G \rightarrow L_{g} G \cong G / \Gamma G$, for any group $G$.

In particular, we have the following result.

Proposition 3.2. Let $\psi: X \rightarrow Y$ be an integral homology equivalence of spaces such that the induced homomorphism $f=\pi_{1} \psi$ is an epimorphism. Then $\operatorname{ker} f \subset \Gamma \pi_{1} X$.

Proof. Since $f$ is an epimorphic $H \mathbb{Z}$-map, we know that ker $f$ is $\pi_{1} X$-perfect.

In other words, for any space $X$, the maximal subgroup that can be factored out of $\pi_{1} X$ without altering the integral homology of $X$, is precisely $\Gamma \pi_{1} X$. In particular, this implies that there is a restriction on the possibility of realizing topologically a universal epimorphic $H \mathbb{Z}$-equivalence of groups (i.e., of finding an integral homology equivalence of spaces that induces localization with respect to a universal epimorphic $H \mathbb{Z}$-equivalence of groups on the fundamental group). 
Proposition 3.3. Let $\mathcal{E}(f)$ be a universal epimorphic $H \mathbb{Z}$-equivalence of groups. Then the following assertions are equivalent:

(i) there exists an integral homology equivalence $\psi: X \rightarrow Y$ of spaces such that $\pi_{1} \psi=\mathcal{E}(f)$ and $L_{\psi}$ is $\pi_{1}$-compatible;

(ii) $\operatorname{ker}\left(l_{H \mathbb{Z}}: G \rightarrow G_{H \mathbb{Z}}\right)=\Gamma G$ for all groups $G$.

Proof. To see that (i) implies (ii), it suffices to show that $\operatorname{ker} l_{H \mathbb{Z}} \subset \Gamma G$. However, this is an immediate consequence of Proposition 3.2, since for every group $G$ we have

$$
L_{\pi_{1} \psi} G \cong L_{\mathcal{E}(f)} G \cong G / \operatorname{ker} l_{H \mathbb{Z}}
$$

Finally, (ii) implies (i), as is shown by our construction of $\varphi$ in (2).

\section{REFERENCES}

[1] A. J. Berrick and C. Casacuberta, A universal space for plus-constructions, Topology 38 (1999), 467-477. MR 2000a:55023

[2] A. K. Bousfield, The localization of spaces with respect to homology, Topology 14 (1975), 133-150. MR 52:1676

[3] A. K. Bousfield, Homological localization towers for groups and П-modules, Memoirs of the American Mathematical Society 10, no. 186 (1977). MR 56:5688

[4] A. K. Bousfield, Homotopical localizations of spaces, American Journal of Mathematics 119 (1997), 1321-1354. MR 98m:55009

[5] C. Casacuberta, Anderson localization from a modern point of view, in: The Čech Centennial, Contemporary Math. 181, Amer. Math. Soc., Providence, RI, 1995, pp. 35-44. MR 96a:55017

[6] C. Casacuberta, J. L. Rodríguez, and Jin-Yen Tai, Localizations of abelian EilenbergMac Lane spaces of finite type, preprint (1998).

[7] C. Casacuberta, J. L. Rodríguez, and D. Scevenels, Singly generated radicals associated with varieties of groups, in: Proceedings of Groups St. Andrews 1997 in Bath I, London Math. Soc., Lecture Note Series 260, Cambridge University Press, Cambridge, 1999. MR 2000h:20044

[8] E. Dror Farjoun, Cellular Spaces, Null Spaces and Homotopy Localization, Lecture Notes in Math. vol. 1622, Springer-Verlag, Berlin, Heidelberg, New York, 1996. MR 98f:55010

[9] E. Dror Farjoun, K. Orr, and S. Shelah, Bousfield localization as an algebraic closure of groups, Israel Journal of Mathematics 66 (1989), 143-153. MR 90j:55016

[10] A. Heller, On the homotopy theory of topogenic groups and groupoids, Illinois Journal of Mathematics 24 (1980), 576-605. MR 83c:55017.

[11] D. J. S. Robinson, A course in the theory of groups, 2nd edition, Graduate Texts in Mathematics, vol. 80, Springer-Verlag, Berlin, Heidelberg, New York, 1996. MR 96f:20001

[12] J. L. Rodríguez and D. Scevenels, Universal epimorphic equivalences for group localizations, Journal of Pure and Applied Algebra 148 (3) (2000), 309-316. MR 2002b:55020

Área de Geometría y Topología, Cite III, Universidad de Almería, E-04120 La Cañada de San Urbano, Almería, Spain

E-mail address: jlrodri@ual.es

$U R L$ : http://www.ual.es/personal/jlrodri

Departement Wiskunde, Katholieke Universiteit Leuven Celestijnenlaan 200 B, B-3001 Heverlee, Belgium

E-mail address: dirk.scevenels@wis.kuleuven.ac.be 